\title{
A retrospective analysis of the follicle-stimulating hormone starting dose in expected normal responders undergoing their first in vitro fertilization cycle: proposed dose versus empiric dose
}

\author{
Dayong Lee ${ }^{1,2}$, Soo Jin Han³, Seul Ki Kim ${ }^{1,2}$, Byung Chul Jee ${ }^{1,2}$ \\ 'Department of Obstetrics and Gynecology, Seoul National University Bundang Hospital, Seongnam; 'Department of Obstetrics and Gynecology, \\ Seoul National University College of Medicine, Seoul; ${ }^{3}$ Department of Obstetrics and Gynecology, Seoul National University Hospital, Seoul, Korea
}

Objective: The purpose of this retrospective study was to evaluate the appropriateness of various follicle-stimulating hormone (FSH) starting doses in expected normal responders based on the nomogram developed by La Marca et al.

Methods: A total of 117 first in vitro fertilization cycles performed from 2011 to 2017 were selected. All women were expected normal responders and used a recombinant FSH and flexible gonadotropin-releasing hormone antagonist protocol. The FSH starting dose was empirically determined $(150,225$, or $300 \mathrm{IU})$. The FSH starting dose indicated by La Marca's nomogram was determined using female age and serum anti-Müllerian hormone or basal FSH levels. If the administered dose was exactly the same as the proposed dose, the cycle was assigned to the concordant group (34 cycles). If not, it was assigned to the discordant group (83 cycles). Optimal ovarian response was defined as a total of $8-14$ oocytes, hypo-response as $<8$ oocytes, and hyper-response as $>14$ oocytes.

Results: Between the concordant and discordant group, ovarian response (optimal, 32.4\% vs. 27.7\%; hypo-response, $55.9 \%$ vs. 54.2\%; and hyper-response, $11.8 \%$ vs. $18.1 \%$ ) and the number of total or mature oocytes were similar. Ovarian hyperstimulation syndrome was rare in both groups ( $0 \%$ vs. $1.2 \%$ ). The implantation rate, clinical pregnancy rate, miscarriage rate, and live birth rate were all similar.

Conclusion: The use of the proposed FSH starting dose determined using La Marca's nomogram did not enhance the optimal ovarian response rate or pregnancy rate in expected normal responders. Individualization of the FSH starting dose by La Marca's nomogram appears to have no distinct advantages over empiric choice of the dose in expected normal responders.

Keywords: Follicle-stimulating hormone; In vitro fertilization; Nomograms; Ovarian hyperstimulation syndrome

\section{Introduction}

When performing ovarian stimulation for in vitro fertilization (IVF), acquiring an appropriate number of oocytes is an important prereq-

Received: Jun 27, 2018 · Revised: Aug 8, 2018 · Accepted: Aug 16, 2018 Corresponding author: Byung Chul Jee

Department of Obstetrics and Gynecology, Seoul National University Bundang Hospital, 82 Gumi-ro 173beon-gil, Bundang-gu, Seongnam 13620, Korea Tel: +82-31-787-7254 Fax:+82-31-787-4054 E-mail: blasto@snubh.org

This is an Open Access article distributed under the terms of the Creative Commons Attribution Non-Commercial License (http://creativecommons.org/licenses/by-nc/4.0/) which permits unrestricted non-commercial use, distribution, and reproduction in any medium, provided the original work is properly cited. uisite for a successful pregnancy. Several previous studies have shown that obtaining 8-14 oocytes is optimal for the likelihood of a successful pregnancy, while avoiding the risk of ovarian hyperstimulation syndrome (OHSS) [1-3]. In women who are expected to be normal responders and are undergoing IVF for the first time, clinicians usually choose $150 \mathrm{IU}$ or $225 \mathrm{IU}$, and sometimes $300 \mathrm{IU}$, of exogenous follicle-stimulating hormone (FSH). This empiric choice is commonly based on a comprehensive consideration of the woman's age and several ovarian reserve tests, such as serum levels of antiMüllerian hormone (AMH) or FSH and the antral follicle count (AFC) [4-9]. The empiric choice of the FSH starting dose can sometimes un- 
expectedly result in a nonoptimal ovarian response.

La Marca et al. [10] proposed a nomogram for individualization of the FSH starting dose. The nomogram proposed by the authors allows the FSH starting dose to be chosen by a combination of female age and serum AMH or FSH. This nomogram was originally developed with the goal of maximizing the likelihood that women who are expected normal responders would obtain eight oocytes. However, a recent study has shown that the optimal number of oocytes, in terms of the live birth rate, was 13 [11]. In addition, it has been suggested that aiming to collect between 18 and 20 oocytes is the most favorable in terms of the cumulative live birth rate [12].

Many clinicians feel that the FSH dose suggested by the nomogram may be lower than expected empirically. An empiric choice of the FSH starting dose can sometimes result in hyper-response; however, if up to 10 oocytes are obtained, blastocyst transfer can be considered to maximize the implantation rate and to decrease the likelihood of a multi-fetal pregnancy. Otherwise, surplus embryos can be frozen and be used in subsequent cycles, maximizing the pregnancy rate.

La Marca et al. [10] developed their nomogram without considering the pregnancy rate. Although the nomogram was validated by a randomized study, that study still did not consider the pregnancy rate [13]. For this reason, their nomogram needs to be further validated. To evaluate the appropriateness of their nomogram, we retrospectively analyzed 117 consecutive first IVF cycles performed in expected normal responders. The FSH starting dose was determined by empiric choice, but it was incidentally concordant with the dose specified by La Marca's nomogram in 34 cycles (the concordant group). The FSH starting dose was not concordant with the dose specified by La Marca's nomogram in the remaining 83 cycles (the discordant group). Cycle outcomes and the pregnancy rate were compared between the two groups.

\section{Methods}

\section{Subjects}

A retrospective study was performed under approval by the Institutional Review Board (IRB No. B-1805/471-101). A total of 117 infertile women who underwent their first IVF cycles from 2011 to 2017 at Seoul National University Bundang Hospital were selected. The selection criteria were the same as in La Marca's study: (1) first IVF or intracytoplasmic sperm injection (ICSI) cycle, (2) female age $\leq 40$ years, (3) regular menstrual cycle of 25-35 days, (4) continuation of the initial starting FSH dose for the first 5 days, (5) serum AMH 1.0-4.0 ng/mL, (6) basal serum $\mathrm{FSH} \leq 15 \mathrm{IU} / \mathrm{L}$, and (7) normal uterine cavity. The exclusion criteria were as follows: (1) polycystic ovarian syndrome, (2) previous ovarian surgery, (3) stage III-IV endometriosis, (4) history of oral contraceptives in the previous 3 months, (5) any proven meta- bolic or endocrine diseases, and (6) frozen embryo transfer.

\section{IVF protocol}

In all cases, recombinant FSH (Gonal F; Merck Serono, Darmstadt, Germany) and a flexible gonadotropin-releasing hormone (GnRH) antagonist protocol were used. The starting dose of recombinant FSH was empirically determined by clinicians (150, 225, or 300 IU). The $\mathrm{GnRH}$ antagonist (Cetrotide, Merck Serono) was administrated daily when the leading follicle reached $14 \mathrm{~mm}$ in diameter. When the leading follicles reached $19 \mathrm{~mm}$ in diameter, ovulation was triggered by injecting $250 \mu \mathrm{g}$ of recombinant human chorionic gonadotropin (Ovidrel, Merck Serono). The oocyte was retrieved 35-36 hours later. Embryo transfer was performed 3 or 5 days after oocyte retrieval. The luteal phase was supported either by daily progesterone injection or vaginal gel (Crinone, Merck Serono). A serum human chorionic gonadotropin test was performed 14 days after oocyte retrieval. The implantation rate was defined as the number of gestational sacs observed on ultrasonography at 6 weeks of pregnancy divided by the number of embryos transferred. Clinical pregnancy was confirmed by the visualization of at least 1 gestational sac. Ongoing pregnancy was defined when the pregnancy had completed $\geq 20$ weeks of gestation. Cleavage-stage grade $A$ embryos were identified on day 3 according to the following morphological criteria: equal-sized blastomeres and no fragments, with no apparent morphologic abnormalities.

\section{Concordance of FSH starting dose}

In the 117 IVF cycles, the proposed FSH starting dose was determined using female age and serum AMH or basal FSH as indicated in La Marca's nomogram. In our hospital, the method of the serum AMH assay changed during the study period (2011 to 2017). The Immunotech (IOT) assay was used until August 2012, the original Gen II kit was used from September 2012 to July 2013, and then the revised Gen II kit was used from August 2013 to July 2016. Since August 2016, we have used a fully automated AMH assay system based on the revised Gen II kit. To correct for these changes, we used the formulas reported in previous studies. The revised Gen II assay results were converted to the original Gen II assay values using the following formula: revised protocol $\mathrm{AMH}$ value $=0.929+1.658 \times$ (original $\mathrm{AMH}$ value) $-0.006 \times$ (original $A M H$ value $)^{2}$ [14]. Subsequently, the original Gen II assay results were converted to the IOT assay values by the following equation: Gen II AMH value $=1.353 \times($ IOT $\mathrm{AMH}$ value $)+0.051$ [15].

If the dose that was administered was exactly the same as the dose specified by the nomogram, cycles were assigned to the concordant group (34 cycles). If not, they were assigned to the discordant group (83 cycles). If the proposed dose was over $225 \mathrm{IU}$, and the administered dose was $300 \mathrm{IU}$, the cycle was assigned to the concordant 
group. If the proposed dose was over $225 \mathrm{IU}$ and the administered dose was 225 or $150 \mathrm{IU}$, the cycle was assigned to the discordant group.

\section{Outcomes and statistical analysis}

In the present study, optimal ovarian response was defined as between 8 and 14 total retrieved oocytes. Hypo-response was defined as fewer than eight retrieved oocytes, and hyper-response was defined as more than 14 retrieved oocytes. All statistical analyses were performed using IBM SPSS ver. 23.0 (IBM Corp., Armonk, NY, USA). Most of the numerical data did not show a normal distribution; thus, the data are presented as median and interquartile range. The nonparametric Mann-Whitney $U$-test was used to compare mean values between groups. The chi-square test or the Fisher exact test was used to compare proportions. The results were considered statistically significant when the $p$-value was $<0.05$.

\section{Results}

The clinical characteristics of both groups are shown in Table 1. Women's age, basal serum FSH levels, and serum AMH levels were all similar between the two groups. The stimulation outcomes are summarized in Table 2. The most frequent actual starting dose was $225 \mathrm{IU}$ (95 cycles) and $28.4 \%$ of those cycles were in the concordant group. In 13 cycles, the starting dose of FSH was $150 \mathrm{IU}$, and most of those cycles were in the discordant group. In nine cycles, $300 \mathrm{IU}$ was used as the starting dose of FSH, and $66.7 \%$ of those cycles were in the concordant group. In the concordant group, the FSH dose was not increased on day 6 of stimulation in any patients, but it was decreased in two women on day 6 of stimulation (Table 2). In the discordant group, the FSH dose was increased starting on day 6 of stimulation in one woman and was decreased on day 6 of stimulation in seven women. Overall, $91.5 \%$ of all cycles maintained the FSH starting dose throughout the entire period of ovarian stimulation.

The total FSH dose administered and the serum estradiol level on triggering day were similar between the concordant and discordant groups (Table 2). The number of total or mature oocytes retrieved, the optimal ovarian response rate, and the rate of hypo-response or

Table 1. Clinical characteristics between the two groups

\begin{tabular}{lccc}
\hline Variable & $\begin{array}{c}\text { Concordant group } \\
\text { (34 cycles) }\end{array}$ & $\begin{array}{c}\text { Discordant group } \\
\text { (83 cycles) }\end{array}$ & $p$-value \\
\hline Age of female (yr) & $34(32-36.3)$ & $34(32-36)$ & 0.981 \\
Age of husband $(\mathrm{yr})$ & $37.5(35-40.3)$ & $37(35-39)$ & 0.523 \\
Parous woman & $9(26.5)$ & $15(18.1)$ & 0.307 \\
Body mass index $\left(\mathrm{kg} / \mathrm{m}^{2}\right)$ & $21.4(20.3-23.2)$ & $21.4(20.3-23.2)$ & 0.777 \\
Cause of infertility & & & - \\
$\quad$ Unexplained & $25(73.5)$ & $43(51.8)$ & \\
Male & $4(11.8)$ & $21(25.3)$ & \\
Tubal & $4(11.8)$ & $15(18.1)$ & \\
$\quad$ Endometriosis & $1(2.9)$ & $4(4.8)$ & \\
Basal serum FSH (IU/L) & $4.8(3.6-6.3)$ & $5.6(4.5-7.4)$ & 0.103 \\
Serum AMH $(\mathrm{ng} / \mathrm{mL})$ & $1.54(1.11-2.09)$ & $1.91(1.17-2.83)$ & 0.059 \\
\hline
\end{tabular}

Values are presented as median (interquartile range) or number (\%). $\mathrm{FSH}$, follicle-stimulating hormone; $\mathrm{AMH}$, anti-Müllerian hormone.

Table 2. Ovarian stimulation outcomes between the two groups

\begin{tabular}{lcc}
\hline Variable & Concordant group (34 cycles) & Discordant group (83 cycles) \\
\hline FSH starting dose (IU) & & $12(14.5)$ \\
150 & $1(2.9)$ & $68(81.9)$ \\
225 & $27(79.4)$ & $3(3.6)$ \\
300 & $6(17.6)$ & $36(43.4)$ \\
More than proposed dose & - & $47(56.6)$ \\
Less than proposed dose & - & 1 \\
FSH dose increased at stimulation day 6 & 0 & 7 \\
FSH dose decreased at stimulation day 6 & 2 & $8(7-9)$ \\
Stimulation day & $7(7-8.3)$ & $1,575(1,350-1,800)$ \\
Total FSH dose (IU) & $1,575(1,575-2,138)$ & $1,264(793-2,622)$ \\
Serum estradiol at triggering day (pg/mL) & $1,445(818-2,350)$ & $7(4-13)$ \\
No. of total oocyte retrieved & $7(4.8-10.3)$ & $45(54.2)$ \\
Hypo-response $(<8)$ & $19(55.9)$ & $23(27.7)$ \\
Optimal response (8-14) & $11(32.4)$ & $15(18.1)$ \\
Hyper-response $(>14)$ & $4(11.8)$ & $4(2-6.3)$ \\
No. of mature oocytes & $3(2-7)$ & - \\
Fertilization rate (\%) & $100(68-100)$ & $0.397(63-100)$
\end{tabular}

Values are presented as number (\%) or median (interquartile range).

$\mathrm{FSH}$, follicle-stimulating hormone. 
Table 3. Embryo transfer and pregnancy outcomes between the two groups

\begin{tabular}{lcc}
\hline Variable & Concordant group (34 cycles) & Discordant group (83 cycles) \\
\hline Cancellation of embryo transfer & 4 & 11 \\
No oocyte & 1 & 1 \\
Failed fertilization & 1 & 4 \\
Arrested embryo development & 0 & 3 \\
All embryo cryopreservation due to OHSS risk or occurrence & 0 & 2 \\
All embryo cryopreservation due to thin endometrium & 2 & 1 \\
Moderate to severe OHSS & 0 & 72 \\
Embryo transfer cycle & 30 & 61 \\
Day 3 transfer & 25 & 11 \\
Day 5 transfer & 5 & $2(2-2)$ \\
No. of embryo transferred & $2(2-2)$ & $1(0-2)$ \\
No. of A grade embryo at day 3 transfer & $1(0-2)$ & $3(2-5)^{b)}$ \\
Surplus embryo cryopreserved & $2(2-3)^{\mathrm{a}}$ & - \\
Implantation rate (\%, $n$ ) & $28.3(15 / 53)$ & $25.0(32 / 128)$ \\
Clinical pregnancy per transfer & $10(33.3)$ & $28(38.9)$ \\
Ongoing pregnancy per transfer & $8(26.7)$ & $23(31.9)$ \\
Miscarriage per clinical pregnancy & $2(20.0)$ & $5(17.9)$ \\
Live birth per transfer & $8(26.7)$ & $23(31.9)$
\end{tabular}

Values are presented as median (interquartile range) or number (\%) unless otherwise indicated. OHSS, ovarian hyperstimulation syndrome.

a) Seven cycles; ${ }^{\text {b) }}$ ifteen cycles.

hyper-response were all similar between the concordant and discordant groups (Table 2).

Embryo transfer and pregnancy outcomes are presented in Table 3. Embryo transfer was cancelled in 15 cycles. A freeze-all policy was implemented due to OHSS risk in two women in the discordant group (serum estradiol levels on triggering day of $3,174 \mathrm{pg} / \mathrm{mL}$ and $2,924 \mathrm{pg} / \mathrm{mL}$ with a total number of 15 and 12 oocytes, respectively). Only one woman (in the discordant group) was hospitalized due to the development of moderate OHSS. There were no significant differences in the implantation rate, clinical pregnancy rate, ongoing pregnancy rate, miscarriage rate, or live birth rate (Table 3).

\section{Discussion}

In this retrospective study, we observed no differences in the optimal ovarian response, acquisition of high-quality embryos, or resultant pregnancy rates according to the concordance of the FSH dose with that calculated by La Marca's nomogram. Efforts have been made to tailor or individualize the FSH starting dose to avoid unfavorable excessive or suboptimal ovarian response. Several nomograms based on the AFC have been proposed to determine individualized FSH starting doses [4,5,16-18]. Those researchers argued that determining the FSH starting dose by those nomograms and subsequent dose adjustment could reduce cycle cancellation due to inadequate ovarian response. However, not enough evidence has emerged to support those nomograms based on the AFC. Individualized FSH dosing did not show any significant benefit when considering the cost-effectiveness of conventional dosing based on $150 \mathrm{IU}$ $[17,19]$.

La Marca et al. [10] proposed a nomogram based on women's age and serum AMH and/or FSH levels. They proposed that using this nomogram would allow an optimal number of oocytes to be obtained in $29.7 \%$ of women even though an FSH dose less than $225 \mathrm{IU}$ was used as the starting dose. Furthermore, it was expected that this would increase the live birth rate and reduce the overall treatment cost. In order to validate La Marca's nomogram, that research group conducted two consecutive studies. First, Papaleo et al. [20] retrospectively analyzed data obtained from two separate institutions. However, they included poor and high responders along with normal responders. La Marca et al. [10] developed their nomogram for normal responders only. Among the 255 women who obtained a suboptimal number of oocytes ( $<8$ or $>14$ ), $18.8 \%$ used an inappropriate FSH dose. They did not present the total number of patients who received each FSH dose or pregnancy outcomes.

Second, Allegra et al. [13] performed a randomized trial to validate the nomogram. They only included expected normal responders. In the patients in whom the FSH starting dose was chosen empirically, it was $150 \mathrm{IU}$ for women $\leq 35$ years and $225 \mathrm{IU}$ for women $>35$ years. They showed a lower rate of optimal ovarian response in the empiric choice group versus the nomogram-based choice group 
(42.4\% vs. 63.0\%). However, the FSH starting dose was $150 \mathrm{IU}$ in $55 \%$ of the women in the empiric choice group, meaning that a suboptimal ovarian response would be expected in that group.

In the present retrospective study, the FSH starting dose was $225 \mathrm{IU}$ in $81.9 \%$ of the women in the discordant dose group. In the study by Papaleo et al. [20], the proportion of women who used $225 \mathrm{IU}$ as the starting dose of FSH in the discordant dose group was similar to our study. In the study by Allegra et al. [13], most women in the empiric choice group used a lower-than-usual dose.

In addition, both of the above studies had limitations in the $\mathrm{AMH}$ assay method. La Marca et al. [10] used the IOT assay, although both validation studies used revised Gen II assay kit values. AMH concentrations using the Gen II assay tend to be higher than those obtained using the IOT, and the revised GEN II results have been reported to be higher than the original GEN II values [14,15]. Therefore, performing a validation study using $\mathrm{AMH}$ results that did not compensate for these differences could have been a significant source of bias in the study results. In this study, AMH values were modified to reflect these differences, which is an advantage over previous studies.

In fact, La Marca's nomogram was developed with the basic goal of avoiding ovarian hyper-response in normal responders. However, in the present retrospective study, the hyper-response rate was similar between the concordant and discordant dose groups although the majority of women received $225 \mathrm{IU}$. Surplus embryos to be cryopreserved were obtained in seven of the 34 cycles in the concordant group $(20.6 \%)$ and in 15 of the 83 cycles in the discordant group (18.1\%). The overall ovarian response was similar between the concordant and discordant dose groups, as was the resultant pregnancy rate. In normal responders, avoidance of ovarian hyper-response may be unnecessary. If up to 10 oocytes are obtained, blastocyst transfer can be considered to maximize the implantation rate and to reduce the likelihood of a multi-fetal pregnancy. Otherwise, surplus embryos can be frozen for use in subsequent thawed cycles, thereby maximizing the pregnancy rate [21].

Therefore, an FSH starting dose of $225 \mathrm{IU}$ would be a good option when considering the cumulative pregnancy rate through consecutive frozen embryo transfer [22]. Moreover, many clinicians increase the FSH starting dose in the second IVF cycle in women who use 150 IU in the first cycle and do not become pregnant. This strategy may result in a more favorable ovarian response and pregnancy outcomes [23]. Many clinicians do not choose $300 \mathrm{IU}$ as an FSH starting dose in normal responders, because severe OHSS should be avoided [24].

This study had some limitations. First, this was not a prospective validation study. Second, women who used a different dose during the first 5 days were excluded. More variable FSH dose schedules exist, such as $225 \mathrm{IU}$ in the first 3 days, with changes thereafter according to women's response. In conclusion, the use of La Marca's nomo- gram seems to have no significant advantages over the conventional empiric choice in terms of the number of oocytes retrieved or the pregnancy outcomes when applied to expected normal responders in IVF/ICSI cycles. Further prospective studies are needed to clarify the advantages of individualized FSH dosing.

\section{Conflict of interest}

No potential conflict of interest relevant to this article was reported.

\section{References}

1. Verberg MF, Eijkemans MJ, Macklon NS, Heijnen EM, Baart EB, Hohmann FP, et al. The clinical significance of the retrieval of a low number of oocytes following mild ovarian stimulation for IVF: a meta-analysis. Hum Reprod Update 2009;15:5-12.

2. Sunkara SK, Rittenberg V, Raine-Fenning N, Bhattacharya S, Zamora J, Coomarasamy A. Association between the number of eggs and live birth in IVF treatment: an analysis of 400135 treatment cycles. Hum Reprod 2011;26:1768-74.

3. Sunkara SK, La Marca A, Seed PT, Khalaf Y. Increased risk of preterm birth and low birthweight with very high number of oocytes following IVF: an analysis of 65868 singleton live birth outcomes. Hum Reprod 2015;30:1473-80.

4. Popovic-Todorovic B, Loft A, Lindhard A, Bangsboll S, Andersson AM, Andersen AN. A prospective study of predictive factors of ovarian response in 'standard' IVF/ICSI patients treated with recombinant FSH: a suggestion for a recombinant FSH dosage normogram. Hum Reprod 2003;18:781-7.

5. Howles CM, Saunders H, Alam V, Engrand P; FSH Treatment Guidelines Clinical Panel. Predictive factors and a corresponding treatment algorithm for controlled ovarian stimulation in patients treated with recombinant human follicle stimulating hormone (follitropin alfa) during assisted reproduction technology (ART) procedures: an analysis of 1378 patients. Curr Med Res Opin 2006;22:907-18.

6. Ledger WL. Clinical utility of measurement of anti-Mullerian hormone in reproductive endocrinology. J Clin Endocrinol Metab 2010;95:5144-54.

7. Al-Azemi M, Killick SR, Duffy S, Pye C, Refaat B, Hill N, et al. Multimarker assessment of ovarian reserve predicts oocyte yield after ovulation induction. Hum Reprod 2011;26:414-22.

8. Lan VT, Linh NK, Tuong HM, Wong PC, Howles CM. Anti-Mullerian hormone versus antral follicle count for defining the starting dose of FSH. Reprod Biomed Online 2013;27:390-9.

9. Moon KY, Kim H, Lee JY, Lee JR, Jee BC, Suh CS, et al. Nomogram to predict the number of oocytes retrieved in controlled ovarian 
stimulation. Clin Exp Reprod Med 2016;43:112-8.

10. La Marca A, Papaleo E, Grisendi V, Argento C, Giulini S, Volpe A. Development of a nomogram based on markers of ovarian reserve for the individualisation of the follicle-stimulating hormone starting dose in in vitro fertilisation cycles. BJOG 2012;119: 1171-9.

11. Polyzos NP, Drakopoulos P, Parra J, Pellicer A, Tournaye $H$, Bosch E, et al. Cumulative live birth rates following the first ovarian stimulation for IVF/ICSI: a European multicenter analysis of 15,000 women using individual patient data. Hum Reprod 2017;32(Suppl 1):i79.

12. Magnusson A, Kallen K, Thurin-Kjellberg A, Bergh C. The number of oocytes retrieved during IVF: a balance between efficacy and safety. Hum Reprod 2018;33:58-64.

13. Allegra A, Marino A, Volpes A, Coffaro F, Scaglione P, Gullo S, et al. $A$ randomized controlled trial investigating the use of a predictive nomogram for the selection of the FSH starting dose in IVF/ ICSI cycles. Reprod Biomed Online 2017;34:429-38.

14. Bonifacio M, Bradley CK, Karia S, Livingstone M, Bowman MC, McArthur SJ. The original Beckman Coulter Generation II assay significantly underestimates AMH levels compared with the revised protocol. J Assist Reprod Genet 2015;32:1691-6.

15. Li HW, Ng EH, Wong BP, Anderson RA, Ho PC, Yeung WS. Correlation between three assay systems for anti-Mullerian hormone (AMH) determination. J Assist Reprod Genet 2012;29:1443-6.

16. Olivennes F, Howles CM, Borini A, Germond M, Trew G, Wikland $M$, et al. Individualizing FSH dose for assisted reproduction using a novel algorithm: the CONSORT study. Reprod Biomed Online 2009;18:195-204.

17. Nyboe Andersen A, Nelson SM, Fauser BC, Garcia-Velasco JA, Klein BM, Arce JC, et al. Individualized versus conventional ovarian stimulation for in vitro fertilization: a multicenter, randomized, controlled, assessor-blinded, phase 3 noninferiority trial. Fertil Steril 2017;107:387-96.e4.
18. van Tilborg TC, Oudshoorn SC, Eijkemans MJ, Mochtar MH, van Golde RJ, Hoek A, et al. Individualized FSH dosing based on ovarian reserve testing in women starting IVF/ICSI: a multicentre trial and cost-effectiveness analysis. Hum Reprod 2017;32:2485-95.

19. van Tilborg TC, Torrance HL, Oudshoorn SC, Eijkemans MJ, Koks CA, Verhoeve HR, et al. Individualized versus standard FSH dosing in women starting IVF/ICSI: an RCT. Part 1: the predicted poor responder. Hum Reprod 2017;32:2496-505.

20. Papaleo E, Zaffagnini S, Munaretto M, Vanni VS, Rebonato G, Grisendi V, et al. Clinical application of a nomogram based on age, serum FSH and AMH to select the FSH starting dose in IVF/ ICSI cycles: a retrospective two-centres study. Eur J Obstet Gynecol Reprod Biol 2016;207:94-9.

21. Roque M, Lattes K, Serra S, Sola I, Geber S, Carreras R, et al. Fresh embryo transfer versus frozen embryo transfer in in vitro fertilization cycles: a systematic review and meta-analysis. Fertil Steril 2013;99:156-62.

22. Sterrenburg MD, Veltman-Verhulst SM, Eijkemans MJ, Hughes EG, Macklon NS, Broekmans FJ, et al. Clinical outcomes in relation to the daily dose of recombinant follicle-stimulating hormone for ovarian stimulation in in vitro fertilization in presumed normal responders younger than 39 years: a meta-analysis. Hum Reprod Update 2011;17:184-96.

23. Popovic-Todorovic B, Loft A, Ziebe S, Andersen AN. Impact of recombinant FSH dose adjustments on ovarian response in the second treatment cycle with IVF or ICSI in "standard" patients treated with $150 \mathrm{lU} /$ day during the first cycle. Acta Obstet Gynecol Scand 2004;83:842-9.

24. Jayaprakasan K, Hopkisson J, Campbell B, Johnson I, Thornton J, Raine-Fenning N. A randomised controlled trial of 300 versus $225 \mathrm{IU}$ recombinant $\mathrm{FSH}$ for ovarian stimulation in predicted normal responders by antral follicle count. BJOG 2010;117:85362. 